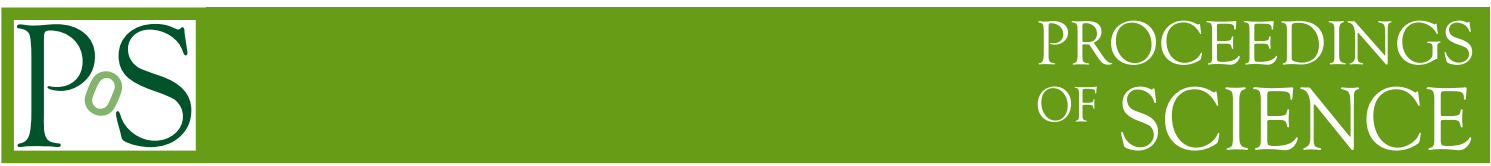

\title{
KM3NeT/ARCA Expectations for the Low Latitude Bubbles
}

\author{
The KM3NeT Collaboration \\ \$ https://www.km3net.org/km3net-author-list-for-icrc-2019 \\ E-mail: antonio.marinelli@pi.infn.it
}

In 2010 it was pointed out that the Fermi-LAT telescope registered high-energy gamma-ray emission from giant outflows symmetric respect to the Galactic plane which exceeded the expected backgrounds. The region were this emission was observed, extending up to $9 \mathrm{kpc}$ from the Galactic plane and covering a total solid angle of $\sim 1 \mathrm{sr}$, was afterward called: Fermi Bubbles. This observation could be interpreted as the trace of an outburst activity of the central supermassive black hole and the collected gamma rays were attributed to the non-thermal emission from cosmic rays accelerated in this region. Since this gamma-ray observations several hypotheses were made about the leptonic or hadronic origin of this emission. In 2016 a new analysis by the HAWC collaboration provided stringent upper limits on the gamma-ray flux from the Northern Fermi Bubble in the energy range of teens of TeV. These limits represent a constrain also for a possible neutrino counterpart making more difficult the observation through high-energy neutrino telescopes. However, in 2017, a study of the Fermi-LAT collaboration focused on the Fermi Bubbles region below 10 degrees of latitude produced the observation of a different spectral behaviour. The resulting spectral energy distribution is harder than the one obtained from the whole Fermi Bubbles. This allows for better observational possibilities for neutrino telescopes. Here we show the expectations for KM3NeT/ARCA.

\footnotetext{
Corresponding authors: Antonio Marinelli ${ }^{1}$, Rosa Coniglione ${ }^{\dagger 2}$, Ankur Sharma ${ }^{1}$

${ }^{1}$ University of Pisa and INFN, Pisa

2 INFN-LNS, Catania
}

36th International Cosmic Ray Conference -ICRC2019-

July 24th - August 1st, 2019

Madison, WI, U.S.A.

\footnotetext{
*for collaboration list see PoS(ICRC2019)1177

${ }^{\dagger}$ Speaker.
} 


\section{Introduction}

In 2014 the Fermi-LAT collaboration confirms a significant gamma-ray emission connected to the Galactic symmetric giant outflows already observed on microwaves, X-rays and polarized radio emission extending up to $9 \mathrm{kpc}$ from the Galactic plane [1,2], the so-called region of Fermi Bubbles. Several studies have been carried out considering its morphology over different wavelengths, still allowing both hadronic and leptonic models $[3,4]$. In order to explain the gamma-ray emission as a product of interaction of cosmic rays (CR) with nuclei of gas in the region, a high-energy neutrino counterpart is also expected [5, 6, 7]. In 2017 the HAWC collaboration presented a new study dedicated to the Northern bubble and poses stringent upper limits on the possible gamma-ray emission above $1 \mathrm{TeV}$ [8], setting a maximal cutoff for the high-energy emission at around $30 \mathrm{TeV}$. Assuming that the southern Bubble behaves like the northern one, these observations pose strong constrains on the possibility to observe a significant high-energy neutrino excess from this region of the sky. The Fermi-LAT analysis published in 2014 excludes the Low Latitude Bubbles (LLB) region $\left(|b|>10^{\circ}\right)$ in order to avoid the flux contamination of point-like sources and molecular clouds present in the Central Molecular Zone (CMZ) [2]. However in 2017 a new paper from the Fermi-LAT, dedicated to dark matter searches, shows a new analysis, mostly referring to the portion of the Bubbles with Galactic coordinates $|l|<15^{\circ}$ and $|b|<10^{\circ}$ [9], the LLB region. Unexpectedly this analysis shows a spectral energy distribution (SED) harder than the one obtained for the entire Bubbles region and a cutoff that can be extended up to hundred of TeVs. If we assume this SED directly linked to the initial wind of the Fermi Bubbles, where cosmic rays (CR) are accelerated, a exciting scenario can be expected for the neutrino emission in the LLB. An analysis presented in 2018 [10] shows that this hard SED cannot be just a underestimation of the diffuse Galactic emission expected in the region of the LLB, suggesting the interaction of accelerating hadrons with the molecular gas as the main physical process for the production of the observed gamma rays. Assuming this scenario for the LLB we compute the expected neutrino SED and the long term expectations for KM3NeT/ARCA neutrino telescope, the cubic kilometer telescope under construction in the ionian sea [11]. In particular we show the observation capabilities of one building block of ARCA for different cutoff assumptions on the LLB SED taking into account 5 years of observation. First estimates for a different detector layout with a bigger volume (about 6 $\mathrm{Km}^{3}$ ) and a different SED assumption were presented in ref. [12].

\section{Low Latitude Fermi Bubbles emission}

The Fermi Bubbles are identified as a symmetric giant outflow structure that from the center of the Milky Way reach a elongation of about $9 \mathrm{kpc}$ to the north and to the south directions perpendicular respect to the Galactic plane [2]. Already observed as a microwave (20-40 GHz) and polarized radio $(2-20 \mathrm{GHz})$ emitters, in 2014 they were reported also as a gamma-ray emitters by the Fermi-LAT collaboration. Whether the origin of this high-energy emission is mainly due to the interaction of accelerated cosmic rays with the nuclei of the gas or to the inverse Compton emission from the accelerated electrons [13] is still under debate. In 2017 the HAWC experiment defines better the SED of the Fermi Bubbles with new high-energy upper limits measured from the Northern Bubble. These upper limits constrain the SED at a few tenths of TeV suggesting a maximal cutoff 
at about $30 \mathrm{TeV}$ for gamma rays, with an equivalent maximal energy for the produced neutrinos at around $15 \mathrm{TeV}$. Considering the position of the Fermi Bubbles these upper limits severely reduce the possibility of neutrino observations with the IceCube neutrino telescope, while leaving some chances for the observation using the next-generation neutrino telescope KM3NeT. Interestingly a new SED measurement from the inner Fermi Bubbles $|l|<15^{\circ}$ and $|b|<10^{\circ}$ comes out from a Fermi-LAT work dedicated to dark matter searches showing a different spectral behavior. This new measurement presents a harder spectrum for the LLB region and shifts the compatible cutoff of corresponding neutrino SED between 100 to $300 \mathrm{TeV}$ s. The neutrino SED showed on the left plot of Fig. 1 is obtained considering the gamma-ray SED that fit the Fermi-LAT and accounting for the reported spectral points of IceCube. The IceCube spectral points reported in Fig. 2 are obtained considering the HESE events spatially correlated with the entire Fermi Bubbles region and after rescaled for the region of the sky covered by the LLB as in [4]. The gamma-ray observations are considered fully hadronic setting as a main production mechanism the interaction of accelerated protons (in the bubble wind) with the molecular gas present in this region of the Galaxy. Therefore the expected neutrino SED is obtained considering the proton-proton interaction, following [14] parametrization for the link between gamma-ray and neutrino fluxes. For this particular study only $v_{\mu}+\bar{v}_{\mu}$ were considered, obtaining a normalization flux $\Phi_{0}=1.5 \times 10^{-6} \mathrm{GeV}_{c m^{-2}} \mathrm{~s}^{-1} \mathrm{sr}^{-1}$ and a spectral index $\alpha=2.1$ with variable cutoff between 100 and $300 \mathrm{TeVs}$.
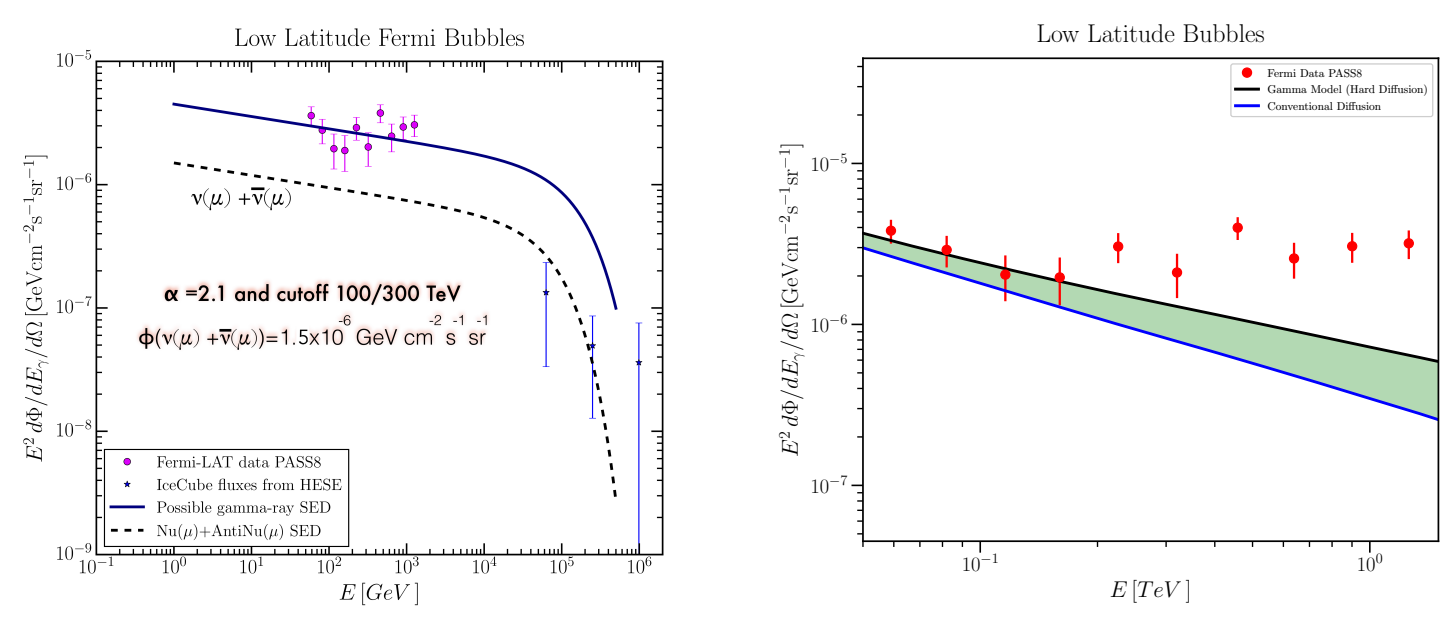

Figure 1: Left) In this plot the SED of the LLB obtained considering the PASS8 data sample [2], reported with magenta points, and the IceCube fluxes limits obtained considering the HESE events compatible with the entire Fermi Bubbles region [4] (blue points). The muonic neutrino SED (with the black dashed line) is obtained considering the Fermi-LAT gamma-ray SED entirely produced by the interaction of CRs with gas. Right) In this plot we report the Fermi-LAT gamma-ray observations for the low latitude Bubbles $\left(\theta<10^{\circ}\right)$ in comparison with the expected diffuse Galactic emission [10]. The green region represent the space of gamma-ray SEDs produced in the same region of the sky (LLB) from the interaction of CR with the gas, assuming different models. The Gamma Model (black line) [15] and conventional diffusion model (blue line).

\section{The KM3NeT neutrino observatory}

The KM3NeT collaboration is building a deep sea infrastructure in the Mediterranean Sea [16]. 
The infrastructure comprises two deep-sea Cherenkov detectors named ARCA and ORCA [11]. The ARCA (Astroparticle Research with Cosmics in the Abyss) detector is deployed $80 \mathrm{~km}$ offshore of Sicily, Italy, at a depth of about $3450 \mathrm{~m}$ and the main objective is the discovery and subsequent observation of TeV-PeV astrophysical neutrino sources. The ORCA (Oscillation Research with Cosmics in the Abyss) detector is deployed $40 \mathrm{~km}$ offshore Toulon, France, at a depth of about $2450 \mathrm{~m}$ with a main objective of neutrino mass ordering determination looking at the oscillation of $\mathrm{GeV}$ atmospheric neutrinos. The main detector component of ARCA is a Digital Optical Module (DOM), a pressure-resistant glass sphere housing 31 3-inch photo-multiplier tubes (PMTs) and their associated electronics. The DOMs are arranged in string-like structures, called Detection Units (DUs), anchored to the seabed and held vertically by the buoyancy of the DOMs as well as a buoy. For ARCA the vertical spacing between the DOMs along a DU is $36 \mathrm{~m}$ while the horizontal spacing between the DUs is $90 \mathrm{~m}$. In the final configuration ARCA will consist of two Building Blocks, each of these composed by 115 DUs, and instrumenting a total volume of $1 \mathrm{~km}^{3}$ of seawater.

\section{Monte Carlo Analysis}

This analysis uses a recent Monte Carlo (MC) production of one building block (115 DUs) of KM3NeT/ARCA for atmospheric neutrinos, neutrino from the LLB and atmospheric muons. Only track-like events were used. Both the charge current (CC) and the neutral current (NC) interactions of (anti-) $v_{\mu}$ and (anti-) $v_{e}$ were considered for atmospheric and LLB source neutrinos. The simulation chain used for this analysis is similar to the one described in [11]. The simulated events, with muons and neutrinos respectively generated through MUPAGE [17] and GENHEN [18], are reconstructed with a track reconstruction code. Beside the reconstructed track parameters, as the direction and the energy of the leptonic track, the code provides some additional "quality" parameters that are correlated with the quality of the fit. The atmospheric neutrino flux simulated is based on the Honda model for the conventional component and the Enberg model for the prompt component. A correction for the knee in CR was applied [11]. The LLB neutrino considered in this analysis are computed considering a source with a $E^{-2.1} \mathrm{SED}$ and the flux normalization obtained from the gamma-ray data of Fig. 1. The final number of events, for both source (LLB) and background, are obtained in terms of events/block/year.

\subsection{Events selection}

The analysis method followed in this paper is similar to that one presented in [12] .To reject the atmospheric muon backgrounds cuts on the variables provided by the reconstruction code are applied. The key variables used for this analysis are:

1. $\theta$ fit: The zenith distribution of the source and background events

2. Reconstructed Galactic coordinates: reconstructed tracks reported in Galactic coordinates.

3. Likelihood: Likelihood given by the track fit procedure $(\lambda)$

4. $\log _{10}(\beta)$ : Logarithm in base 10 of the error estimate on the fit calculated as $\sqrt{e r r_{v x}^{2} \oplus e r r_{v y}^{2}}$, where $e r r_{v x}$ and $e r r_{v y}$ are the error estimated for the $\mathrm{x}$ and $\mathrm{y}$ versors of the reconstructed track in the reference system where the track is on the $\mathrm{z}$ axis 


\section{5. $\log _{10}\left(\mathbf{E}_{r e c}\right):$ Logarithm in base 10 of the reconstructed energy}

A preselection of the events was done selecting events in the region of LLB $\left(\left|l_{\text {rec }}\right|<15^{\circ}\right.$ and $\left.\left|b_{\text {rec }}\right|<10^{\circ}\right)$ and upgoing tracks $\left(\theta f i t>90^{\circ}\right)$. The number of events per year after the preselection cuts are reported in Tab 1.

To obtain the best sensitivity ( $90 \%$ of confidence level) and the discovery potential ( $5 \sigma$ in the $50 \%$ of the pseudo experiment) reported in Fig. 2 we optimize the cuts on Likelihood, $\log _{10}(\beta)$ and $E_{\text {rec }}$ parameters to produce the higher signal to noise ratio.

The cuts on $\lambda$ and $\beta$ remove most of the atmospheric muon badly reconstructed as up-going while $\theta$ fit cut on the reconstructed energy remove mainly the atmospheric background. These cuts were simultaneously determined.

The final cuts that we obtain for the reported parameters to optimize the signal to noise ratio are the follow: $\theta$ fit $<90^{\circ}, \lambda>260, \log _{10}(\beta)>-2.0$, and $E_{\text {rec }}>4 \mathrm{TeV}$ for the case of a neutrino SED with cutoff at $100 \mathrm{TeV}$, while $E_{\text {rec }}>25 \mathrm{TeV}$ for the case of a neutrino SED with a cutoff at $300 \mathrm{TeV}$.

In table 1 we report the number of events of signal and background at the preliminary cuts level and after the final cuts.

\section{Results and discussion}

From the range of parameters reported in the previous section we proceed to find the ones who give us the best values of discovery potential (at $5 \sigma$ level) and sensitivity ( $90 \%$ confidence level) for the case of a LLB SED with a cutoff of $100 \mathrm{TeV}$ and a SED with a cutoff at $300 \mathrm{TeV}$. These values are considered here as the minimum and the maximum cutoff compatible with the PASS8 spectral points as well as the IceCube spectral points obtained from the HESE catalog. As discussed in Section 2 the expected neutrino SED was obtained considering the gamma-ray emission measured by Fermi-LAT as fully produced by the interaction of CRs in the initial part of the Fermi Bubbles with the ambient gas. It is worth mentioning that the assumption of a gamma-ray SED dominated by an Inverse Compton processes, due to accelerated electrons, would have changed completely the results. A further update of this analysis foresees including also the shower reconstruction analysis and using templated morphologies of LLB with the gamma-ray emission as extracted from FermiLAT data, which will improve the analysis sensitivity. For the region of LLB we did not account for the HAWC upper limits since this region is not considered in the recent HAWC analysis, mainly because outside of the experiment field of view. The HAWC upper limits exclusion allows to fix the cutoff of expected neutrino SED at higher energies respect to the entire Fermi Bubbles, increasing the signal to noise ratio for this particular region. More informations about the possible cutoff to apply to the neutrino SED can arise when considering the samples of astrophysical neutrinos at lower energies beside the HESE events.

\section{Conclusions}

In this analysis we show the long term expectations of the future KM3NeT/ARCA neutrino telescope for the inner regions (LLB) of the already known Fermi Bubbles. Interestingly, considering the hard SED and a possible cutoff at hundreds of TeV, this region can be considered a optimal target 


\begin{tabular}{|l|l|l|l|l|}
\hline \hline SED & cuts level & $v_{\text {sig }}$ & $v_{\text {atm }}$ & $\mu_{\text {atm }}$ \\
\hline$E^{-2} e^{-E / 100 T e V}$ & Prel. Cuts Level & 71 & 857 & $2.45 \times 10^{4}$ \\
$E^{-2} e^{-E / 100 T e V}$ & Final Cuts Level & 39 & 38 & 0 \\
$E^{-2} e^{-E / 300 T e V}$ & Prel. Cuts Level & 83 & 857 & $2.45 \times 10^{4}$ \\
$E^{-2} e^{-E / 300 T e V}$ & Final Cuts Level & 29 & 6 & 0 \\
\hline
\end{tabular}

Table 1: Obtained events. In table we report the reconstructed track-like events/ARCA Building Block/year for the signal and the background after the preliminary cuts of the LLB direction $\left(\left|l_{\text {rec }}\right|<15^{\circ}\right.$ and $\left.\left|b_{r e c}\right|<10^{\circ}\right)$ and $\theta$ fit $<90^{\circ}$, and after the final cuts: $\theta$ fit $<90^{\circ}, \lambda>260, \log _{10}(\beta)>-2.0$, and $E_{r e c}>4 \mathrm{TeV}$ for the $v_{\mu}+\bar{v}_{\mu}$ Cutoff at $100 \mathrm{TeV}$, while $E_{\text {rec }}>25 \mathrm{TeV}$ for the $v_{\mu}+\bar{v}_{\mu}$ Cutoff at $300 \mathrm{TeV}$.
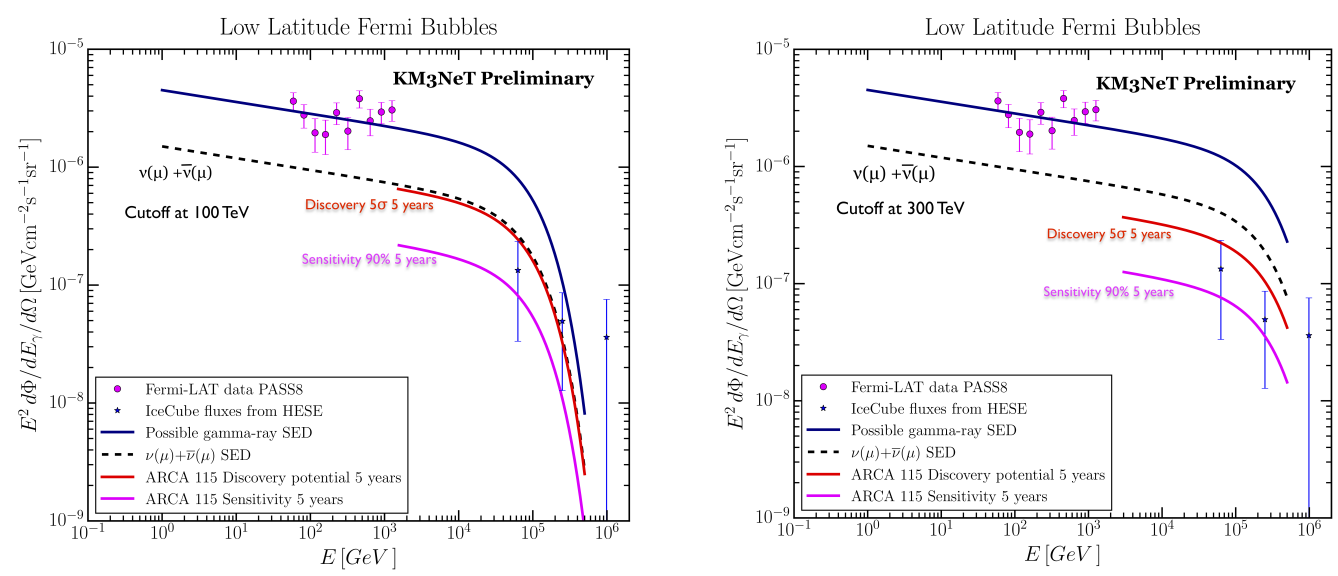

Figure 2: a) In this plot we report the Sensitivity $90 \% \mathrm{CL}$ and the Discovery potential $(5 \sigma)$ for one building block of ARCA telescope (115 DUs ) considering the muonic neutrino + antineutrino SED expected from the LLB region with a Cutoff of $100 \mathrm{TeV}$. The expectations are reported for a reconstructed energy above 4 $\mathrm{TeV}$, as suggested by the optimal energy cut obtained.

b) In this plot we report the sensitivity $90 \% \mathrm{CL}$ and the discovery potential $(5 \sigma)$ for one building block of ARCA telescope (115 DUs ) considering the muonic neutrino SED expected from the LLBubbles SED with a Cutoff of $300 \mathrm{TeV}$. The expectations are reported for a reconstructed energy above $25 \mathrm{TeV}$, as suggested by the optimal energy cut obtained.

for the incoming ARCA experiment. We highlight the fact that this new SED, obtained considering the Fermi-LAT data, cannot be just an underestimation of diffuse Galactic component present in this region of the sky. In fact it was already demonstrated in a recent presentation [10] that the expected diffuse Galactic emission in the LLB region has a different spectral behavior at energies above $100 \mathrm{GeV}$ s. Considering that, here, we link this initial wind part of the Fermi Bubbles (LLB) with a dominant proton population explaining the Fermi-LAT gamma-ray SED as the production of accelerated CR interaction with the gas environment. The corresponding neutrino counterpart will be observable with 5 years of observations of one building block of KM3NeT/ARCA, even considering the case of a pessimistic cutoff at $100 \mathrm{TeV}$. 


\section{References}

[1] M. Su, T. R. Slatyer, and D. P. Finkbeiner, "Giant Gamma-ray Bubbles from Fermi-LAT: Active Galactic Nucleus Activity or Bipolar Galactic Wind?, the Astrophysical Journal, vol. 724, pp. 1044-1082, Dec 2010.

[2] M. Ackermann, A. Albert, W. B. Atwood, L. Baldini, and et al., "The Spectrum and Morphology of the Fermi Bubbles," the Astrophysical Journal, vol. 793, p. 64, Sept. 2014.

[3] R. M. Crocker, G. V. Bicknell, A. M. Taylor, and E. Carretti, “A Unified Model of the Fermi Bubbles, Microwave Haze, and Polarized Radio Lobes: Reverse Shocks in the Galactic Centers Giant Outflows," the Astrophysical Journal, vol. 808, p. 107, Aug 2015.

[4] C. Lunardini, S. Razzaque, and L. Yang, "Multimessenger study of the fermi bubbles: Very high energy gamma rays and neutrinos," Physical Review D, vol. 92, p. 021301, Jul 2015.

[5] S. Razzaque and L. Yang, "Hadronic Models of the Fermi Bubbles: Future Perspectives," Galaxies, vol. 6, p. 47, Apr 2018.

[6] K. Fang, M. Su, T. Linden, and K. Murase, "IceCube and HAWC constraints on very-high-energy emission from the Fermi bubbles,” Physical Review D, vol. 96, p. 123007, Dec 2017.

[7] N. Sherf, U. Keshet, and I. Gurwich, "IceCube Constraints on the Fermi Bubbles," the Astrophysical Journal, vol. 847, p. 95, Oct 2017.

[8] A. U. Abeysekara, A. Albert, R. Alfaro, C. Alvarez, J. D. Álvarez, R. Arceo, J. C. Arteaga-Velázquez, H. A. Ayala Solares, A. S. Barber, N. Bautista-Elivar, A. Becerril, E. Belmont-Moreno, and et al., "Search for Very High-energy Gamma Rays from the Northern Fermi Bubble Region with HAWC," the Astrophysical Journal, vol. 842, p. 85, Jun 2017.

[9] M. Ackermann, M. Ajello, A. Albert, W. B. Atwood, L. Baldini, J. Ballet, G. Barbiellini, D. Bastieri, R. Bellazzini, E. Bissaldi, R. D. Bland ford, E. D. Bloom, and Fermi LAT Collaboration, "The Fermi Galactic Center GeV Excess and Implications for Dark Matter," the Astrophyscial Journal, vol. 840, p. 43, May 2017.

[10] A. Marinelli, D. Grasso, and S. Ventura, "Towards an anagraphical picture of high-energy Galactic neutrinos," in European Physical Journal Web of Conferences, vol. 209 of European Physical Journal Web of Conferences, p. 01003, Sep 2019.

[11] S. Adrián-Martínez, M. Ageron, F. Aharonian, S. Aiello, A. Albert, F. Ameli, E. Anassontzis, M. Andre, G. Androulakis, M. Anghinolfi, and et al., "Letter of intent for KM3NeT 2.0," Journal of Physics G Nuclear Physics, vol. 43, p. 084001, Aug 2016.

[12] KM3NeT Collaboration, S. Adrián-Martínez, M. Ageron, J. A. Aguilar, F. Aharonian, S. Aiello, A. Albert, M. Alexandri, F. Ameli, E. G. Anassontzis, and et al., "Detection potential of the KM3NeT detector for high-energy neutrinos from the Fermi bubbles," Astroparticle Physics, vol. 42, pp. 7-14, Feb 2013.

[13] G. Dobler, D. P. Finkbeiner, I. Cholis, T. Slatyer, and N. Weiner, “The Fermi Haze: A Gamma-ray Counterpart to the Microwave Haze," the Astrophysical Journal, vol. 717, pp. 825-842, Jul 2010.

[14] F. L. Villante and F. Vissani, "How precisely can neutrino emission from supernova remnants be constrained by gamma ray observations?," Phys. Rev. D, vol. 78, p. 103007, Nov 2008.

[15] D. Gaggero, D. Grasso, A. Marinelli, A. Urbano, and M. Valli, "The Gamma-Ray and Neutrino Sky: A Consistent Picture of Fermi-LAT, Milagro, and IceCube Results," the Astrophysical Journal Letters, vol. 815, p. L25, Dec. 2015. 
[16] S. Aiello, S. E. Akrame, F. Ameli, E. G. Anassontzis, M. Andre, G. Androulakis, M. Anghinolfi, G. Anton, M. Ardid, J. Aublin, and et al., "Sensitivity of the KM3NeT/ARCA neutrino telescope to point-like neutrino sources," Astroparticle Physics, vol. 111, pp. 100-110, Sep 2019.

[17] G. Carminati, M. Bazzotti, A. Margiotta, and M. Spurio, "Atmospheric MUons from PArametric formulas: a fast GEnerator for neutrino telescopes (MUPAGE)," Computer Physics Communications, vol. 179, pp. 915-923, Dec 2008.

[18] C. Distefano, "gSeaGen: A GENIE-based code for neutrino telescopes," in European Physical Journal Web of Conferences, vol. 116 of European Physical Journal Web of Conferences, p. 08001, Apr 2016. 